\title{
Author Index Volume 44 (2015)
}

The issue number is given in front of the pagination

Aalten, P., see König, A. (2) 675-685

Abdollahian, D., see McClure, R. (1) 283-295

Acquaah-Mensah, G.K., N. Agu, T. Khan and A. Gardner, A Regulatory Role for the Insulin- and BDNFLinked RORA in the Hippocampus: Implications for Alzheimer's Disease (3) 827-838

Adlard, P.A., see Robb, E. (1) 69-78

Agu, N., see Acquaah-Mensah, G.K. (3) 827-838

Ahmed, S.H., see Zarrouk, A. (4) 1349-1359

Ajit, D., see Walker, J.M. (2) 561-572

Akuffo, K.O., see Nolan, J.M. (4) 1157-1169

Albert, M., see Nowrangi, M.A. (2) 585-598

Al-khateeb, E., A. Althaher, M. Al-khateeb, H. Al-Musawi, O. Azzouqah, S. Al-Shweiki and Y. Shafagoj, Relation between Uric Acid and Alzheimer's Disease in Elderly Jordanians (3) 859-865

Al-khateeb, M., see Al-khateeb, E. (3) 859-865 Alkon, D.L., see Khan, T.K. (3) 729-744 Ally, B.A., see Molitor, R.J. (1) 1-12

Al-Musawi, H., see Al-khateeb, E. (3) 859-865

Al-Shweiki, S., see Al-khateeb, E. (3) 859-865

Althaher, A., see Al-khateeb, E. (3) 859-865

Althoff, V., see Barucker, C. (2) 613-624

Amano, N., see Okamoto, N. (3) 777-786

Ames, D., see Lazarus, J. (1) 175-182

Ames, D., see Rembach, A. (3) 917-925

Ammar, Z.M., see Foley, A.M. (3) 787-795

Ammou, S.B., see Zarrouk, A. (4) 1349-1359

Ancín, I., see Cuesta, P. (2) 493-505

Ander, B.P., see Zhan, X. (4) 1213-1229

Ando, K., see Frederick, C. (4) 1145-1156

Andrews, S., D. Das, K.J. Anstey and S. Easteal, Interactive Effect of $A P O E$ Genotype and Blood Pressure on Cognitive Decline: The PATH Through Life Study (4) 1087-1098

Ang, C.-S., see Watt, A.D. (4) 1131-1143

Anker, U., see Suppa, P. (1) 183-193

Anstey, K.J., see Andrews, S. (4) 1087-1098

Antón, M., see Ordóñez-Gutiérrez, L. (4) 1063-1068

Arbuckle, M., see Claxton, A. (3) 897-906

Arena, A., see Di Domenico, F., (4) 1107-1120
Arighi, A., see Calvi, A. (1) 277-282

Armstrong, N.J., see Lazarus, J. (1) 175-182

Arnaldi, D., see Morbelli, S. (3) 815-826

Ashby, E.L., see Jochemsen, H.M. (1) 153-162

Atwood, C.S., see Bowen, R.L. (2) 549-560

Auff, E., see Sieczkowski, E. (1) 103-114

Aurtenetxe, S., see Cuesta, P. (2) 493-505

Azzarello, D.M., see Bulati, M. (4) 1241-1251

Azzouqah, O., see Al-khateeb, E. (3) 859-865

Baches, S., see Hüttenrauch, M. (4) 1291-1302

Baek, M.J., see Jang, J.-W. (3) 1023-1034

Baglioni, M., see Mangialasche, F. (2) 649-660

Bajo, R., see Cuesta, P. (2) 493-505

Bakchine, S., see Salza, R. (4) 1253-1261

Baker, L.D., see Claxton, A. (3) 897-906

Bamne, M.N., see Chaudhry, M. (3) 989-994

Barabash, A., see Cuesta, P. (2) 493-505

Barkhof, F., see Möller, C. (2) 635-647

Barnham, K.J., see Robb, E. (1) 69-78

Barnham, K.J., see Watt, A.D. (4) 1131-1143

Barone, E., see Di Domenico, F., (4) 1107-1120

Barrett, J.P., A.M. Minogue, R.S. Jones, C. Ribeiro, R.J. Kelly and M.A. Lynch, Bone Marrow-Derived Macrophages from A $\beta P P / P S 1$ Mice are Sensitized to the Effects of Inflammatory Stimuli (3) 949-962

Barucker, C., A. Sommer, G. Beckmann, M. Eravci, A. Harmeier, C.G. Schipke, D. Brockschnieder, T. Dyrks, V. Althoff, P.E. Fraser, L.-N. Hazrati, P. St George-Hyslop, J.C.S. Breitner, O. Peters and G. Multhaup, Alzheimer Amyloid Peptide $A \beta_{42}$ Regulates Gene Expression of Transcription and Growth Factors (2) 613-624

Bathsavanis, A., see Dauphinot, V. (3) 907-916

Bayer, T.A., see Hüttenrauch, M. (4) 1291-1302

Bayer, T.A., see Sieczkowski, E. (1) 103-114

Beatty, S., see Nolan, J.M. (4) 1157-1169

Beck, S., see Suppa, P. (1) 183-193

Becker, J.T., see Riverol, M. (1) 319-328

Beckmann, G., see Barucker, C. (2) 613-624

Beecham, G., see Humphries, C.E. (3) 977-987 
Beeri, M.S., see Ravona-Springer, R. (2) 687-693

Beeri, M.S., see West, R.K. (3) 927-935

Behl, C., see Claxton, A. (3) 897-906

Behl, C., see Renziehausen, J. (3) 879-896

Bell, S., see Jefferson, A.L. (4) 1361-1373

Bellinger, F.P., see Rueli, R.H.L.H. (2) 379-383

Bellinger, M.T., see Rueli, R.H.L.H. (2) 379-383

Bensadoun, G., see König, A. (2) 675-685

Benson, E.M., see Jefferson, A.L. (4) 1361-1373

Bentham, P., see Wischik, C.M. (2) 705-720

Berge, J., see Eckerström, C. (1) 205-214

Berger, C., A.-K. Erbe, I. Ehlers, I. Marx, K. Hauenstein and S. Teipel, Effects of Task-Irrelevant Emotional Stimuli on Working Memory Processes in Mild Cognitive Impairment (2) 439-453

Bernstein, L.H., see Ingenbleek, Y. (3) 745-754

Berres, M., see Egli, S.C. (2) 625-633

Berry, M.J., see Rueli, R.H.L.H. (2) 379-383

Bickel, H., see Ramirez, A. (4) 1203-1212

Bjerke, M., see Eckerström, C. (1) 205-214

Bladowska, J., see Zimny, A. (1) 329-338

Blankenberg, S., see Tynkkynen, J. (3) 1007-1013

Blankenstein, M.A., see Teunissen, C.E. (3) 809-813

Blarzino, C., see Di Domenico, F., (4) 1107-1120

Blennow, K., see Kettunen, P. (4) 13131322

Blesa, R., see Olde Rikkert, M.G.M. (2) 471-480

Blessing, K., see Kawada, H. (1) 297-307

Bolger, M., see Nolan, J.M. (4) 1157-1169

Bongers, A., see Olde Rikkert, M.G.M. (2) 471-480

Bopp, I., see Suppa, P. (1) 183-193

Borjini, N., see Meunier, J. (2) 507-524

Bossert, I., see Morbelli, S. (3) 815-826

Bottoni, G., see Morbelli, S. (3) 815-826

Bowen, R.L., G. Perry, C. Xiong, M.A. Smith and C.S. Atwood, A Clinical Study of Lupron Depot in the Treatment of Women with Alzheimer's Disease: Preservation of Cognitive Function in Patients Taking an Acetylcholinesterase Inhibitor and Treated with High Dose Lupron Over 48 Weeks (2) 549-560

Bozzali, M., C. Dowling, L. Serra, B. Spanò, M. Torso, C. Marra, D. Castelli, N.G. Dowell, G. Koch, C. Caltagirone and M. Cercignani, The Impact of Cognitive Reserve on Brain Functional Connectivity in Alzheimer's Disease (1) 243-250

Brancorsini, S., see Mangialasche, F. (2) 649-660

Brayne, C., see Wu, Y.-T. (4) 1099-1106

Breitner, J.C.S., see Barucker, C. (2) 613-624

Bremond, F., see König, A. (2) 675-685

Breteler, M.M., see Ramirez, A. (4) 1203-1212

Brettschneider, C., see Ramirez, A. (4) 1203-1212
Brion, J.-P., see Frederick, C. (4) 1145-1156

Brisindi, M., see Costa, A. (1) 63-67

Brockschnieder, D., see Barucker, C. (2) 613-624

Brodaty, H., see Lazarus, J. (1) 175-182

Brugnolo, A., see Morbelli, S. (3) 815-826

Bruña, R., see Cuesta, P. (2) 493-505

Buchert, R., see Suppa, P. (1) 183-193

Buée, L., see Frederick, C. (4) 1145-1156

Buffa, S., see Bulati, M. (4) 1241-1251

Bulati, M., S. Buffa, A. Martorana, F. Gervasi, C. Camarda, D.M. Azzarello, R. Monastero, C. Caruso and G. Colonna-Romano, Double Negative $\left(\mathrm{IgG}^{+} \mathrm{IgD}^{-} \mathrm{CD}_{27}^{-}\right)$B Cells are Increased in a Cohort of Moderate-Severe Alzheimer's Disease Patients and Show a Pro-Inflammatory Trafficking Receptor Phenotype (4) 1241-1251

Burns, A., see Semrau, M. (1) 139-151

Buschiazzo, A., see Morbelli, S. (3) 815-826

Bush, A.I., see Robb, E. (1) 69-78

Butterfield, D.A., see Di Domenico, F., (4) 1107-1120

Cabranes, J.A., see Cuesta, P. (2) 493-505

Cabrera, A.S., see Naranjo, I.C. (2) 695-704

Caffarra, P., see Calvi, A. (1) 277-282

Callaghan, M., see Claxton, A. (3) 897-906

Callaway, J.K., N.C. Jones, A.G. Royse and C.F. Royse, Memory Impairment in Rats after Desflurane Anesthesia is Age and Dose Dependent (3) 995-1005

Caltagirone, C., see Bozzali, M. (1) 243-250

Caltagirone, C., see Costa, A. (1) 63-67

Calvi, A., S.M.G. Cioffi, P. Caffarra, C. Fenoglio, M. Serpente, A.M. Pietroboni, A. Arighi, L. Ghezzi, S. Gardini, E. Scarpini and D. Galimberti, The Novel GRN g.1159_1160delTG Mutation is Associated with Behavioral Variant Frontotemporal Dementia (1) 277-282

Camarda, C., see Bulati, M. (4) 1241-1251

Cao, L., see Zhu, X.-C. (1) 163-174

Capistrant, B.D., see Rist, P.M. (4) 1171-1180

Carey, M., see Graham, S.F. (3) 851-857

Carlesimo, G.A., see Costa, A. (1) 63-67

Carmichael, O.T., see Riverol, M. (1) 319-328

Caruso, C., see Bulati, M. (4) 1241-1251

Castellanos, N.P., see Cuesta, P. (2) 493-505

Castelli, D., see Bozzali, M. (1) 243-250

Cavallaro, R.A., see Persichilli, S. (4) 1323-1331

Cecchetti, R., see Mangialasche, F. (2) 649-660

Cercignani, M., see Bozzali, M. (1) 243-250

Chaabane, W., see Zarrouk, A. (4) 1349-1359

Chala, S.R., see Naranjo, I.C. (2) 695-704

Chan, E.C.Y., see Chang, K.L. (1) 215-231 
Chan, M., see Goh, L.K. (1) 57-61

Chang, K.L., H.N. Pee, W.P. Tan, G.S. Dawe, E. Holmes, J.K. Nicholson, E.C.Y. Chan and P.C. Ho, Metabolic Profiling of CHO-A $\beta \mathrm{PP}_{695}$ Cells Revealed Mitochondrial Dysfunction Prior to Amyloid- $\beta$ Pathology and Potential Therapeutic Effects of Both PPAR $\gamma$ and PPAR $\alpha$ Agonisms for Alzheimer's Disease (1) 215-231

Chang, R.C.-C., see Yu, J. (2) 455-469

Chang, Y.-L., T.-F. Chen, Y.-C. Shih, M.-J. Chiu, S.-H. Yan and W.-Y. Isaac Tseng, Regional Cingulum Disruption, Not Gray Matter Atrophy, Detects Cognitive Changes in Amnestic Mild Cognitive Impairment Subtypes (1) 125-138

Chapman IV, W.G., see Gifford, K.A. (1) 309-318

Chappell, D., see Seeburger, J.L. (2) 525-539

Charles, J., G. Naglie, J. Lee, R. Moineddin, S. Jaglal and M.C. Tierney, Self-Report Measures of WellBeing Predict Incident Harm Due to Self-Neglect in Cognitively Impaired Seniors Who Live Alone (2) $425-430$

Chaudhry, M., X. Wang, M.N. Bamne, S. Hasnain, F.Y. Demirci, O.L. Lopez and M.I. Kamboh, Genetic Variation in Imprinted Genes is Associated with Risk of Late-Onset Alzheimer's Disease (3) 989 994

Chekmenev, E., see McClure, R. (1) 283-295

Chen, C.P., see Mohamed, N.-E. (2) 431-437

Chen, T.-F., see Chang, Y.-L. (1) 125-138

Cherny, R.A., see Robb, E. (1) 69-78

Cherubini, A., see Littlejohns, T.J. (4) 1231-1239

Chin, J., see Kim, Y.J. (4) 1075-1085

Chiu, M.-J., see Chang, Y.-L. (1) 125-138

Cho, H., see Kim, H.J. (3) 963-975

Cho, H., see Kim, Y.J. (4) 1075-1085

Choe, Y.S., see Kim, H.J. (3) 963-975

Choi, S.H., see Park, H.K. (2) 661-673

Cholerton, B., see Claxton, A. (3) 897-906

Chong, M.S., see Goh, L.K. (1) 57-61

Chuen-Chung Chang, R., see Huang, C. (3) 839-850

Chun, Y.S., Y. Park, H.G. Oh, T.-W. Kim, H.O. Yang, M.K. Park and S. Chung, O-GlcNAcylation Promotes Non-Amyloidogenic Processing of Amyloid- $\beta$ Protein Precursor via Inhibition of Endocytosis from the Plasma Membrane (1) 261-275

Chung, S., see Chun, Y.S. (1) 261-275

Chung, S.J., see Kim, Y.J. (4) 1075-1085

Cioff, S.M.G., see Calvi, A. (1) 277-282

Claxton, A., L.D. Baker, A. Hanson, E.H. Trittschuh, B. Cholerton, A. Morgan, M. Callaghan, M. Arbuckle, C. Behl and S. Craft, Long-Acting Intranasal
Insulin Detemir Improves Cognition for Adults with Mild Cognitive Impairment or Early-Stage Alzheimer's Disease Dementia (3) 897-906

Cockayne, N., see Diamond, K. (4) 1181-1191

Coen, R.F., see Nolan, J.M. (4) 1157-1169

Colonna-Romano, G. see Bulati, M. (4) 1241-1251

Combrinck, M., see Seeburger, J.L. (2) 525-539

Combs, C.K., see Puig, K.L. (4) 1263-1278

Costa, A., L. Fadda, R. Perri, M. Brisindi, M.G. Lombardi, C. Caltagirone and G.A. Carlesimo, Sensitivity of a Time-Based Prospective Memory Procedure in the Assessment of Amnestic Mild Cognitive Impairment (1) 63-67

Craft, S., see Claxton, A. (3) 897-906

Crispim Junior, C.F., see König, A. (2) 675-685

Cuenca, J.C.P., see Naranjo, I.C. (2) 695-704

Cuesta, P., P. Garcés, N.P. Castellanos, M.E. López, S. Aurtenetxe, R. Bajo, J.A. Pineda-Pardo, R. Bruña, A.G. Marín, M. Delgado, A. Barabash, I. Ancín, J.A. Cabranes, A. Fernandez, F. del Pozo, M. Sancho, A. Marcos, A. Nakamura and F. Maestú, Influence of the APOE $\varepsilon 4$ Allele and Mild Cognitive Impairment Diagnosis in the Disruption of the MEG Resting State Functional Connectivity in Sources Space (2) 493-505

Dale, A.M., see Tang, X. (2) 599-611

Dallob, A., see Seeburger, J.L. (2) 525-539

Damangir, S., see Vuorinen, M. (1) 93-101

Damon, S.M., see Gifford, K.A. (1) 309-318

Dantu, K., see Ryan, N.S. (4) 1069-10704

Darreh-Shori, T., see Lane, R.M. (4) 1039-1062

Das, D., see Andrews, S. (4) 1087-1098

Dauphinot, V., F. Delphin-Combe, C. Mouchoux, A. Dorey, A. Bathsavanis, Z. Makaroff, I. Rouch and P. Krolak-Salmon, Risk Factors of Caregiver Burden Among Patients with Alzheimer's Disease or Related Disorders: A Cross-Sectional Study (3) 907-916

David, R., see König, A. (2) 675-685

Dawe, G.S., see Chang, K.L. (1) 215-231

De Carli, F., see Morbelli, S. (3) 815-826

de San Juan, B.D., see Naranjo, I.C. (2) 695-704

Debbabi, M., see Zarrouk, A. (4) 1349-1359

DeCarli, C., see Zhan, X. (4) 1213-1229

Dekker, J.M., see Teunissen, C.E. (3) 809-813

del Pozo, F., see Cuesta, P. (2) 493-505

Delgado, M., see Cuesta, P. (2) 493-505

Delphin-Combe, F., see Dauphinot, V. (3) 907-916

Demirci, F.Y., see Chaudhry, M. (3) 989-994

Dennison, J., see Nolan, J.M. (4) 1157-1169 
Derreumaux, A., see König, A. (2) 675-685

Desiderio, C., see Persichilli, S. (4) 1323-1331

Dewing, A.S.T., see Rueli, R.H.L.H. (2) 379-383

Di Domenico, F., G. Pupo, C. Mancuso, E. Barone, F. Paolini, A. Arena, C. Blarzino, F.A. Schmitt, E. Head, D.A. Butterfield and M. Perluigi, Bach1 Overexpression in Down Syndrome Correlates with the Alteration of the HO-1/BVR-A System: Insights for Transition to Alzheimer's Disease (4) 1107-1120

Di Napoli, A., see Persichilli, S. (4) 1323-1331

Diamond, K., L. Mowszowski, N. Cockayne, L. Norrie, M. Paradise, D.F. Hermens, S.J.G. Lewis, I.B. Hickie and S.L. Naismith, Randomized Controlled Trial of a Healthy Brain Ageing Cognitive Training Program: Effects on Memory, Mood, and Sleep (4) 1181-1191

Didic, M., see Morbelli, S. (3) 815-826

Dieleman, N., see Möller, C. (2) 635-647

DiTacchio, K.A., S.F. Heinemann and G. Dziewczapolski, Metformin Treatment Alters Memory Function in a Mouse Model of Alzheimer's Disease (1) $43-48$

Djukic-Dejanovic, S., see Semrau, M. (1) 139-151

Do, K.-A., see Rembach, A. (3) 917-925

Doecke, J.D., see Rembach, A. (3) 917-925

Dorey, A., see Dauphinot, V. (3) 907-916

Doumpoulakis, S., see Zygouris, S. (4) 1333-1347

Dowell, N.G., see Bozzali, M. (1) 243-250

Dowling, C., see Bozzali, M. (1) 243-250

Drzezga, A., see Morbelli, S. (3) 815-826

Duffy, S.L., see Elcombe, E.L. (4) 1279-1290

Duits, A., see Teunissen, C.E. (3) 809-813

Dyrks, T., see Barucker, C. (2) 613-624

Dziewczapolski, G., see DiTacchio, K.A. (1) 43-48

Easteal, S., see Andrews, S. (4) 1087-1098

Eckerström, C., E. Olsson, N. Klasson, J. Berge, A. Nordlund, M. Bjerke and A. Wallin, Multimodal Prediction of Dementia with up to 10 Years Follow Up: The Gothenburg MCI Study (1) 205-214

Efrati, S., see Ravona-Springer, R. (2) 687-693

Egli, S.C., D.I. Hirni, K.I. Taylor, M. Berres, A. Regeniter, A. Gass, A.U. Monsch and M. Sollberger, Varying Strength of Cognitive Markers and Biomarkers to Predict Conversion and Cognitive Decline in an Early-Stage-Enriched Mild Cognitive Impairment Sample (2) 625-633

Ehlers, I., see Berger, C. (2) 439-453

Eichler, T., see Wucherer, D. (3) 1015-1021

Elcombe, E.L., J. Lagopoulos, S.L. Duffy, S.J.G. Lewis, L. Norrie, I.B. Hickie and S.L. Naismith,
Hippocampal Volume in Older Adults at Risk of Cognitive Decline: The Role of Sleep, Vascular Risk, and Depression (4) 1279-1290

Elliott, C.T., see Graham, S.F. (3) 851-857

Eraslan, D., see Semrau, M. (1) 139-151

Eravci, M., see Barucker, C. (2) 613-624

Erbe, A.-K., see Berger, C. (2) 439-453

Esiri, M.M., see Mohamed, N.-E. (2) 431-437

Fabrizi, G.M., see Testi, S. (1) 49-56

Fadda, L., see Costa, A. (1) 63-67

Faux, N.G., see Watt, A.D. (4) 1131-1143

Feng, J., see Zhang, C. (2) 375-378

Fenoglio, C., see Calvi, A. (1) 277-282

Fernandez, A., see Cuesta, P. (2) 493-505

Ferrucci, L., see Littlejohns, T.J. (4) 1231-1239

Finkelstein, D.I., see Robb, E. (1) 69-78

Flynn, M., see Seeburger, J.L. (2) 525-539

Foley, A.M., Z.M. Ammar, R.H. Lee and C.S. Mitchell, Systematic Review of the Relationship between Amyloid- $\beta$ Levels and Measures of Transgenic Mouse Cognitive Deficit in Alzheimer's Disease (3) 787-795

Foster, N.L., see Puig, K.L. (4) 1263-1278

Fowler, C.J., see Watt, A.D. (4) 1131-1143

Fox, N.C., see Ryan, N.S. (4) 1069-10704

Francis, P.T., see Mohamed, N.-E. (2) 431-437

Fraser, P.E., see Barucker, C. (2) 613-624

Frederick, C., K. Ando, K. Leroy, C. Héraud, V. Suain, L. Buée and J.-P. Brion, Rapamycin Ester Analog CCI-779/Temsirolimus Alleviates Tau Pathology and Improves Motor Deficit in Mutant Tau Transgenic Mice (4) 1145-1156

Fried, L., see Riverol, M. (1) 319-328

Frih, M., see Zarrouk, A. (4) 1349-1359

Frisoni, G.B., see Morbelli, S. (3) 815-826

Fuchs, A., see Ramirez, A. (4) 1203-1212

Fuentes, J.M., see Naranjo, I.C. (2) 695-704

Fuso, A., see Persichilli, S. (4) 1323-1331

Gach, H.M., see Riverol, M. (1) 319-328

Galimberti, D., see Calvi, A. (1) 277-282

Galluzzi, S., see Morbelli, S. (3) 815-826

Garcés, P., see Cuesta, P. (2) 493-505

García, A.F., see Naranjo, I.C. (2) 695-704

Gardini, S., see Calvi, A. (1) 277-282

Gardner, A., see Acquaah-Mensah, G.K. (3) 827-838

Gass, A., see Egli, S.C. (2) 625-633

Gearing, M., see Rangaraju, S. (3) 797-808

Geerlings, M.I., see Jochemsen, H.M. (1) 153-162

Gerth, J., see Hüttenrauch, M. (4) 1291-1302

Gervasi, F., see Bulati, M. (4) 1241-1251 
Gervasoni, J., see Persichilli, S. (4) 1323-1331

Ghanbari, H.A.,

Ghezzi, L., see Calvi, A. (1) 277-282

Giakoumis, D., see Zygouris, S. (4) 1333-1347

Giardina, B., see Persichilli, S. (4) 1323-1331

Giera, R., see Sieczkowski, E. (1) 103-114

Gifford, K.A., D. Liu, S.M. Damon, W.G. Chapman IV, R.R. Romano III, L.R. Samuels, Z. Lu and A.L. Jefferson for the Alzheimer's Disease Neuroimaging Initiative, Subjective Memory Complaint Only Relates to Verbal Episodic Memory Performance in Mild Cognitive Impairment (1) 309-318

Gifford, K.A., see Jefferson, A.L. (4) 1361-1373

Gilbert, J., see Humphries, C.E. (3) 977-987

Gillis, C., see Meunier, J. (2) 507-524

Gleeson, L.D., see Piguet, O. (2) 541-547

Glymour, M.M., see Rist, P.M. (4) 1171-1180

Gocke, C., see Suppa, P. (1) 183-193

Goh, L.K., W.S. Lim, S. Teo, A. Vijayaraghavan, M. Chan, L. Tay, T.P. Ng, C.H. Tan, T.S. Lee and M.S. Chong, TOMM40 Alterations in Alzheimer's Disease Over a 2-Year Follow-Up Period (1) 57-61

Gore, J.C., see McClure, R. (1) 283-295

Goyal, A.I., see Hawkins, K.M. (3) 867-878

Graham, S.F., M.B. Nasarauddin, M. Carey, B. McGuinness, C. Holscher, P.G. Kehoe, S. Love, A.P. Passmore, C.T. Elliott, A. Meharg and B.D. Green, Quantitative Measurement of $[\mathrm{Na}+]$ and $[\mathrm{K}+]$ in Postmortem Human Brain Tissue Indicates Disturbances in Subjects with Alzheimer's Disease and Dementia with Lewy Bodies (3) 851-857

Grant, W.B., see Wu, Y.-T. (4) 1099-1106

Green, B.D., see Graham, S.F. (3) 851-857

Guedj, E., see Morbelli, S. (3) 815-826

Guerrero-Berroa, E., see West, R.K. (3) 927-935

Haj-Hassan, S., see Jefferson, A.L. (4) 1361-1373

Hajieva, P., see Renziehausen, J. (3) 879-896

Hall, A., M. Muñoz-Ruiz, J. Mattila, J. Koikkalainen, M. Tsolaki, P. Mecocci, I. Kloszewska, B. Vellas, S. Lovestone, P.J. Visser, J. Lötjonen and H. Soininen, for the Alzheimer Disease Neuroimaging Initiative, the AddNeuroMed consortium, DESCRIPA and Kuopio L-MCI, Generalizability of the Disease State Index Prediction Model for Identifying Patients Progressing from Mild Cognitive Impairment to Alzheimer's Disease (1) 79-92

Hammami, M., see Zarrouk, A. (4) 1349-1359

Hammami, S., see Zarrouk, A. (4) 1349-1359

Hampel, H., see Suppa, P. (1) 183-193

Han, C., see Semrau, M. (1) 139-151

Han, H.J., see Park, H.K. (2) 661-673
Han, J.W., see Yu, S.-Y. (1) 195-204

Han, S.-H., see Park, H.K. (2) 661-673

Hanson, A., see Claxton, A. (3) 897-906

Hao, X.-K., see Wang, H.-F. (1) 115-123

Haratz, S., see Ravona-Springer, R. (2) 687-693

Harmeier, A., see Barucker, C. (2) 613-624

Harrington, C.R., see Wischik, C.M. (2) 705-720

Harrison, J., see Olde Rikkert, M.G.M. (2) 471-480

Hascup, E.R., see Hascup, K.N. (3) 771-776

Hascup, K.N. and E.R. Hascup, Altered Neurotransmission Prior to Cognitive Decline in A $\beta P P / P S 1$ Mice, a Model of Alzheimer's Disease (3) 771776

Hashimoto, A.C., see Rueli, R.H.L.H. (2) 379-383

Hasnain, S., see Chaudhry, M. (3) 989-994

Hauenstein, K., see Berger, C. (2) 439-453

Havulinna, A.S., see Tynkkynen, J. (3) 1007-1013

Hawkins, K.M., A.I. Goyal and L.E. Sergio, Diffusion Tensor Imaging Correlates of Cognitive-Motor Decline in Normal Aging and Increased Alzheimer's Disease Risk (3) 867-878

Haworth, J., see Stothart, G. (2) 397-408

Hazrati, L.-N., see Barucker, C. (2) 613-624

Head, E., see Di Domenico, F., (4) 1107-1120

Heijboer, A.C., see Teunissen, C.E. (3) 809-813

Heinemann, S.F., see DiTacchio, K.A. (1) 43-48

Henley, W.E., see Littlejohns, T.J. (4) 1231-1239

Héraud, C., see Frederick, C. (4) 1145-1156

Hermens, D.F., see Diamond, K. (4) 1181-1191

Hernesniemi, J.A., see Tynkkynen, J. (3) 1007-1013

Hertel, J., see Wucherer, D. (3) 1015-1021

Heymann, A., see West, R.K. (3) 927-935

Hickie, I.B., see Diamond, K. (4) 1181-1191

Hickie, I.B., see Elcombe, E.L. (4) 1279-1290

Hiebel, C., see Renziehausen, J. (3) 879-896

Hijón, C.C., see Naranjo, I.C. (2) 695-704

Hirni, D.I., see Egli, S.C. (2) 625-633

Hisahara, S., see Matsumura, A. (2) 409-423

Ho, P.C., see Chang, K.L. (1) 215-231

Ho, Y.-S., see Huang, C. (3) 839-850

Hodges, J.R., see Piguet, O. (2) 541-547

Hoffman, H., see West, R.K. (3) 927-935

Hoffmann, W., see Wucherer, D. (3) 1015-1021

Höftberger, R., see Sieczkowski, E. (1) 103-114

Hohman, T.J., see Jefferson, A.L. (4) 1361-1373

Holder, D.J., see Seeburger, J.L. (2) 525-539

Holland, D., see Tang, X. (2) 599-611

Holmes, E., see Chang, K.L. (1) 215-231

Holmgren, S., see Kettunen, P. (4) 13131322

Holscher, C., see Graham, S.F. (3) 851-857

Hoon, C., see Piguet, O. (2) 541-547

Howard, A., see Nolan, J.M. (4) 1157-1169 
Howard, R.J., see Marchant, N.L. (3) 755-770

Hua, Z., see Ji, L. (1) 13-25

Huang, C., Y.-S. Ho, O.T.-W. Ng, M.G. Irwin, R. Chuen-Chung Chang and G.T.-C. Wong, Dexmedetomidine Directly Increases Tau Phosphorylation (3) 839-850

Huang, X., see Yu, J. (2) 455-469

Humpel, C., see Koal, T. (4) 1193-1201

Humphries, C.E., M.A. Kohli, L. Nathanson, P. Whitehead, G. Beecham, E. Martin, D.C. Mash, M.A. Pericak-Vance and J. Gilbert, Integrated Whole Transcriptome and DNA Methylation Analysis Identifies Gene Networks Specific to Late-Onset Alzheimer's Disease (3) 977-987

Hung, L.W., see Robb, E. (1) 69-78

Hüttenrauch, M., S. Baches, J. Gerth, T.A. Bayer, S. Weggen and O. Wirths, Neprilysin Deficiency Alters the Neuropathological and Behavioral Phenotype in the 5XFAD Mouse Model of Alzheimer's Disease (4) 1291-1302

Hwang, B.-F., see Jung, C.-R. (2) 573-584

Hyman, B.T., see Saidi, L.-J. (3) 937-947

Im, K., see Kim, H.J. (3) 963-975

Ingenbleek, Y. and L.H. Bernstein, Downsizing of Lean Body Mass is a Key Determinant of Alzheimer's Disease (3) 745-754

Irwin, M.G., see Huang, C. (3) 839-850

Isaac Tseng, W.-Y., see Chang, Y.-L. (1) 125-138

Ivitz, E., see Oláh, Z. (4) 1303-1312

Iwahara, N., see Matsumura, A. (2) 409-423

Jaeger, R., see McClure, R. (1) 283-295

Jaglal, S., see Charles, J. (2) 425-430

Jang, J.-W., S.Y. Park, Y.H. Park, M.J. Baek, J.-S. Lim, Y.C. Youn and S.Y. Kim, A Comprehensive Visual Rating Scale of Brain Magnetic Resonance Imaging: Application in Elderly Subjects with Alzheimer's Disease, Mild Cognitive Impairment, and Normal Cognition (3) 1023-1034

Jang, S.-H., see Yu, S.-Y. (1) 195-204

Janka, Z., see Oláh, Z. (4) 1303-1312

Jefferson, A.L., see Gifford, K.A. (1) 309-318

Jefferson, A.L., T.J. Hohman, D. Liu, S. Haj-Hassan, K.A. Gifford, E.M. Benson, J.S. Skinner, Z. Lu, J. Sparling, E.C. Sumner, S. Bell and F.L. Ruberg, Adverse Vascular Risk is Related to Cognitive Decline in Older Adults (4) 1361-1373

Jeon, S., see Kim, Y.J. (4) 1075-1085

Jessen, F., see Ramirez, A. (4) 1203-1212
Ji, L., X. Zhao and Z. Hua, Potential Therapeutic Implications of Gelsolin in Alzheimer's Disease (1) 13-25

Jiang, T., see Wang, H.-F. (1) 115-123

Jiang, T., see Zhu, X.-C. (1) 163-174

Jickling, G.C., see Zhan, X. (4) 1213-1229

Jin, L.-W., see Rangaraju, S. (3) 797-808

Jin, L.-W., see Zhan, X. (4) 1213-1229

Joachim, C., see Seeburger, J.L. (2) 525-539

Jochemsen, H.M., W.M. van der Flier, E.L. Ashby, C.E. Teunissen, R.E. Jones, M.P. Wattjes, P. Scheltens, M.I. Geerlings, P.G. Kehoe and M. Muller, Angiotensin-Converting Enzyme in Cerebrospinal Fluid and Risk of Brain Atrophy (1) 153-162

Johnstone, D.M., see Stone, J. (2) 355-373

Jones, G., see Woodward, M.C. (1) 233-242

Jones, N.C., see Callaway, J.K. (3) 995-1005

Jones, R.E., see Jochemsen, H.M. (1) 153-162

Jones, R.S., see Barrett, J.P. (3) 949-962

Jung, C.-R., Y.-T. Lin and B.-F. Hwang, Ozone, Particulate Matter, and Newly Diagnosed Alzheimer's Disease: A Population-Based Cohort Study in Taiwan (2) 573-584

Kador, P.F., see Kawada, H. (1) 297-307

Kaduszkiewicz, H., see Ramirez, A. (4) 1203-1212

Kálmán, J., see Oláh, Z. (4) 1303-1312

Kamboh, M.I., see Chaudhry, M. (3) 989-994

Kamphuis, P.J.G.H., see Olde Rikkert, M.G.M. (2) 471480

Kao, P.F., see Zhan, X. (4) 1213-1229

Karagiannidis, C., see Zygouris, S. (4) 1333-1347

Kawada, H., K. Blessing, T. Kiyota, T. Woolman, L. Winchester and P.F. Kador, Effects of Multifunctional Antioxidants on Mitochondrial Dysfunction and Amyloid- $\beta$ Metal Dyshomeostasis (1) 297307

Kawamata, J., see Matsumura, A. (2) 409-423

Kay, K.R., see Saidi, L.-J. (3) 937-947

Kazanina, N., see Stothart, G. (2) 397-408

Kehoe, P.G., see Graham, S.F. (3) 851-857

Kehoe, P.G., see Jochemsen, H.M. (1) 153-162

Kelly, R.J., see Barrett, J.P. (3) 949-962

Kemmler, G., see Koal, T. (4) 1193-1201

Kettunen, P., S. Larsson, S. Holmgren, S. Olsson, L. Minthon, H. Zetterberg, K. Blennow, S. Nilsson and A. Sjölander, Genetic Variants of GSK3B are Associated with Biomarkers for Alzheimer's Disease and Cognitive Function (4) 13131322

Khan, T., see Acquaah-Mensah, G.K. (3) 827-838 
Khan, T.K. and D.L. Alkon, Peripheral Biomarkers of Alzheimer's Disease (3) 729-744

Khundakar, A.A. and A.J. Thomas, Neuropathology of Depression in Alzheimer's Disease: Current Knowledge and the Potential for New Treatments (1) $27-41$

Ki, C.-S., see Kim, Y.J. (4) 1075-1085

Kilimann, I., see Wucherer, D. (3) 1015-1021

Kim, B.C., see Park, H.K. (2) 661-673

Kim, E.-J., see Park, H.K. (2) 661-673

Kim, H.J., K. Im, H. Kwon, J.M. Lee, B.S. Ye, Y.J. Kim, H. Cho, Y.S. Choe, K.H. Lee, S.T. Kim, J.S. Kim, J.H. Lee, D.L. Na and S.W. Seo, Effects of Amyloid and Small Vessel Disease on White Matter Network Disruption (3) 963-975

Kim, H.J., see Kim, Y.J. (4) 1075-1085

Kim, H.J., see Park, H.K. (2) 661-673

Kim, J.-H., see Kim, Y.J. (4) 1075-1085

Kim, J.-H., see Kim, Y.J. (4) 1075-1085

Kim, J.S., see Kim, H.J. (3) 963-975

Kim, K.W., see Yu, S.-Y. (1) 195-204

Kim, S.T., see Kim, H.J. (3) 963-975

Kim, S.T., see Kim, Y.J. (4) 1075-1085

Kim, S.Y., see Jang, J.-W. (3) 1023-1034

Kim, T.H., see Yu, S.-Y. (1) 195-204

Kim, T.-W., see Chun, Y.S. (1) 261-275

Kim, Y.J., H. Cho, Y.J. Kim, C.-S. Ki, S.J. Chung, B.S. Ye, H.J. Kim, J.-H. Kim, S.T. Kim, K.H. Lee, S. Jeon, J.-M. Lee, J. Chin, J.-H. Kim, D.L. Na, J.-K. Seong and S.W. Seo, Apolipoprotein E4 Affects Topographical Changes in Hippocampal and Cortical Atrophy in Alzheimer's Disease Dementia: A Five-Year Longitudinal Study (4) 1075-1085

Kim, Y.J., see Kim, H.J. (3) 963-975

Kim, Y.J., see Kim, Y.J. (4) 1075-1085

Kins, S., see Renziehausen, J. (3) 879-896

Kitamura, Y. see Matsumura, A. (2) 409-423

Kivipelto, M., see Mangialasche, F. (2) 649-660

Kivipelto, M., see Vuorinen, M. (1) 93-101

Kiyota, T., see Kawada, H. (1) 297-307

Klaghofer, R., see Suppa, P. (1) 183-193

Klakotskaia, D., see Walker, J.M. (2) 561-572

Klasson, N., see Eckerström, C. (1) 205-214

Klavins, K., see Koal, T. (4) 1193-1201

Kloszewska, I., see Hall, A. (1) 79-92

Ko, P.C., see Molitor, R.J. (1) 1-12

Koal, T., K. Klavins, D. Seppi, G. Kemmler and C. Humpel, Sphingomyelin SM(d18:1/18:0) is Significantly Enhanced in Cerebrospinal Fluid Samples Dichotomized by Pathological
Amyloid- $\beta_{42}$, Tau, and Phospho-Tau-181 Levels (4) $1193-1201$

Koch, G., see Bozzali, M. (1) 243-250

Kochan, N.A., see Lazarus, J. (1) 175-182

Kögel, D., see Renziehausen, J. (3) 879-896

Kohli, M.A., see Humphries, C.E. (3) 977-987

Koifman, K., see West, R.K. (3) 927-935

Koikkalainen, J., see Hall, A. (1) 79-92

Koktysh, D., see McClure, R. (1) 283-295

König, A., C.F. Crispim Junior, A. Derreumaux, G. Bensadoun, P.-D. Petit, F. Bremond, R. David, F. Verhey, P. Aalten and P. Robert, Validation of an Automatic Video Monitoring System for the Detection of Instrumental Activities of Daily Living in Dementia Patients (2) 675-685

König, H.-H., see Ramirez, A. (4) 1203-1212

Kook, K.A., see Wischik, C.M. (2) 705-720

Kos, K., see Littlejohns, T.J. (4) 1231-1239

Kovacs, G.G., see Sieczkowski, E. (1) 103-114

Krolak-Salmon, P., see Dauphinot, V. (3) 907-916

Kuller, L.H., see Riverol, M. (1) 319-328

Kundu, A., see Renziehausen, J. (3) 879-896

Kurumatani, N., see Okamoto, N. (3) 777-786

Kussmaul, L., see Mangialasche, F. (2) 649-660

Kwok, J.B.J., see Lazarus, J. (1) 175-182

Kwon, H., see Kim, H.J. (3) 963-975

Laatikainen, T., see Tynkkynen, J. (3) 1007-1013

Lagopoulos, J., see Elcombe, E.L. (4) 1279-1290

Lai, M.K.P., see Mohamed, N.-E. (2) 431-437

Lane, R.M. and T. Darreh-Shori, Understanding the Beneficial and Detrimental Effects of Donepezil and Rivastigmine to Improve their Therapeutic Value (4) 1039-1062

Lang, I.A., see Littlejohns, T.J. (4) 1231-1239

Langa, K.M., see Littlejohns, T.J. (4) 1231-1239

Lange, C., see Ramirez, A. (4) 1203-1212

Larsson, S., see Kettunen, P. (4) 13131322

Lashley, T., see Ryan, N.S. (4) 1069-10704

Laterza, O., see Seeburger, J.L. (2) 525-539

Lazarus, J., K.A. Mather, N.J. Armstrong, F. Song, A. Poljak, A. Thalamuthu, T. Lee, N.A. Kochan, H. Brodaty, M.J. Wright, D. Ames, P.S. Sachdev and J.B.J. Kwok, DNA Methylation in the Apolipoprotein-A1 Gene is Associated with Episodic Memory Performance in Healthy Older Individuals (1) 175-182

Lecic-Tosevski, D., see Semrau, M. (1) 139-151

Lee, H.-y., see Wu, Y.-T. (4) 1099-1106

Lee, J., see Charles, J. (2) 425-430

Lee, J.H., see Kim, H.J. (3) 963-975 
Lee, J.H., see Mohamed, N.-E. (2) 431-437

Lee, J.-H., see Park, H.K. (2) 661-673

Lee, J.M., see Kim, H.J. (3) 963-975

Lee, J.-M., see Kim, Y.J. (4) 1075-1085

Lee, K.H., see Kim, H.J. (3) 963-975

Lee, K.H., see Kim, Y.J. (4) 1075-1085

Lee, R.H., see Foley, A.M. (3) 787-795

Lee, T., see Lazarus, J. (1) 175-182

Lee, T.-J., see Yu, S.-Y. (1) 195-204

Lee, T.S., see Goh, L.K. (1) 57-61

Lee, Y., see Park, H.K. (2) 661-673

Leroith, D., see West, R.K. (3) 927-935

Leroy, K., see Frederick, C. (4) 1145-1156

Leszek, J., see Zimny, A. (1) 329-338

Levey, A., see Rangaraju, S. (3) 797-808

Lewis, S.J.G., see Diamond, K. (4) 1181-1191

Lewis, S.J.G., see Elcombe, E.L. (4) 1279-1290

Leyton, C.E., see Piguet, O. (2) 541-547

Li, X., see Yu, J. (2) 455-469

Liberski, P.P., see Sieczkowski, E. (1) 103-114

Lim, J.-S., see Jang, J.-W. (3) 1023-1034

Lim, W.S., see Goh, L.K. (1) 57-61

Lin, Y.-T., see Jung, C.-R. (2) 573-584

Littlejohns, T.J., K. Kos, W.E. Henley, A. Cherubini, L. Ferrucci, I.A. Lang, K.M. Langa, D. Melzer and D.J. Llewellyn, Serum Leptin and Risk of Cognitive Decline in Elderly Italians (4) 12311239

Liu, D., see Gifford, K.A. (1) 309-318

Liu, D., see Jefferson, A.L. (4) 1361-1373

Liu, D., see Zhan, X. (4) 1213-1229

Liu, J., see Yu, J. (2) 455-469

Liu, X., K. Ye and D. Weinshenker, Norepinephrine Protects against Amyloid- $\beta$ Toxicity via TrkB (1) 251-260

Liu, Y., see Wang, H.-F. (1) 115-123

Lizard, G., see Zarrouk, A. (4) 1349-1359

Llewellyn, D.J., see Littlejohns, T.J. (4) 1231-1239

Lobo, A., see Semrau, M. (1) 139-151

Lombardi, M.G., see Costa, A. (1) 63-67

Longstreth Jr., W.T., see Riverol, M. (1) 319-328

Lopera, F., see MacPherson, S.E. (2) 481-492

López, M.E., see Cuesta, P. (2) 493-505

Lopez, O.L. see Chaudhry, M. (3) 989-994

López, O.L., see Riverol, M. (1) 319-328

Loskutova, E., see Nolan, J.M. (4) 1157-1169

Lötjonen, J., see Hall, A. (1) 79-92

Love, S., see Graham, S.F. (3) 851-857

Lovestone, S., see Hall, A. (1) 79-92

Lu, Z., see Gifford, K.A. (1) 309-318

Lu, Z., see Jefferson, A.L. (4) 1361-1373
Luck, T., see Ramirez, A. (4) 1203-1212

Luo, X., see Yu, J. (2) 455-469

Lutz, B.M., see Puig, K.L. (4) 1263-1278

Lyketsos, C., see Nowrangi, M.A. (2) 585-598

Lynch, M.A., see Barrett, J.P. (3) 949-962

Ma, Q., see Yu, J. (2) 455-469

Macaulay, S.L., see Rembach, A. (3) 917-925

Macioszek, A., see Zimny, A. (1) 329-338

MacPherson, S.E., M.A. Parra, S. Moreno, F. Lopera and S.D. Sala, Dual Memory Task Impairment in E280A Presenilin-1 Mutation Carriers (2) 481-492

Maestú, F., see Cuesta, P. (2) 493-505

Maier, W., see Ramirez, A. (4) 1203-1212

Makaroff, Z., see Dauphinot, V. (3) 907-916

Mancuso, C., see Di Domenico, F., (4) 1107-1120

Mandelkow, E.-M., see Saidi, L.-J. (3) 937-947

Mangialasche, F., M. Baglioni, R. Cecchetti, M. Kivipelto, C. Ruggiero, D. Piobbico, L. Kussmaul, R. Monastero, S. Brancorsini and P. Mecocci, Lymphocytic Mitochondrial Aconitase Activity is Reduced in Alzheimer's Disease and Mild Cognitive Impairment (2) 649-660

Manocha, G.D., see Puig, K.L. (4) 1263-1278

Maquart, F.-X., see Salza, R. (4) 1253-1261

Marchant, N.L. and R.J. Howard, Cognitive Debt and Alzheimer's Disease (3) 755-770

Marcos, A., see Cuesta, P. (2) 493-505

Marden, J.R., see Rist, P.M. (4) 1171-1180

Marín, A.G., see Cuesta, P. (2) 493-505

Marra, C., see Bozzali, M. (1) 243-250

Martin, E., see Humphries, C.E. (3) 977-987

Martins, R.N., see Rembach, A. (3) 917-925

Martorana, A., see Bulati, M. (4) 1241-1251

Marx, I., see Berger, C. (2) 439-453

Mash, D.C., see Humphries, C.E. (3) 977-987

Masters, C.L., see Rembach, A. (3) 917-925

Masters, C.L., see Robb, E. (1) 69-78

Masters, C.L., see Watt, A.D. (4) 1131-1143

Mather, K.A., see Lazarus, J. (1) 175-182

Matsumura, A., S. Suzuki, N. Iwahara, S. Hisahara, J. Kawamata, H. Suzuki, A. Yamauchi, K. Takata, Y. Kitamura and S. Shimohama, Temporal Changes of CD68 and $\alpha 7$ Nicotinic Acetylcholine Receptor Expression in Microglia in Alzheimer's DiseaseLike Mouse Models (2) 409-423

Mattila, J., see Hall, A. (1) 79-92

Maurice, T., see Meunier, J. (2) 507-524

McClure, R., D. Yanagisawa, D. Stec, D. Abdollahian, D. Koktysh, D. Xhillari, R. Jaeger, G. Stanwood, E. Chekmenev, I. Tooyama, J.C. Gore and W. 
Pham, Inhalable Curcumin: Offering the Potential for Translation to Imaging and Treatment of Alzheimer's Disease (1) 283-295

McGuinness, B., see Graham, S.F. (3) 851-857

Mecocci, P., see Hall, A. (1) 79-92

Mecocci, P., see Mangialasche, F. (2) 649-660

Meharg, A., see Graham, S.F. (3) 851-857

Melzer, D., see Littlejohns, T.J. (4) 1231-1239

Meng, X.-F., see Wang, J. (2) 385-396

Meng, X.-F., see Zhu, X.-C. (1) 163-174

Merril, C.R.

Merril, C.R. and H.A. Ghanbari, Two Hundred Issues Later: The Dream Comes True (3) 727-728

Meunier, J., N. Borjini, C. Gillis, V. Villard and T. Maurice, Brain Toxicity and Inflammation Induced In Vivo in Mice by the Amyloid- $\beta$ FortyTwo Inducer Aftin-4, a Roscovitine Derivative (2) 507-524

Michalowsky, B., see Wucherer, D. (3) 1015-1021

Mielke, M.M., see Nowrangi, M.A. (2) 585-598

Mihai, A., see Semrau, M. (1) 139-151

Milenkovic, I., see Sieczkowski, E. (1) 103-114

Miller, M.I., see Tang, X. (2) 599-611

Minogue, A.M., see Barrett, J.P. (3) 949-962

Minthon, L., see Kettunen, P. (4) 13131322

Mitchell, C.S., see Foley, A.M. (3) 787-795

Mitrofanis, J., see Stone, J. (2) 355-373

Modur, V., see Seeburger, J.L. (2) 525-539

Mohamed, N.-E., J.H. Lee, P.T. Francis, M.M. Esiri, C.P. Chen and M.K.P. Lai, Differential Alterations of Neocortical GluN Receptor Subunits in Patients with Mixed Subcortical Ischemic Vascular Dementia and Alzheimer's Disease (2) 431-437

Moineddin, R., see Charles, J. (2) 425-430

Molitor, R.J., P.C. Ko and B.A. Ally, Eye Movements in Alzheimer's Disease (1) 1-12

Möller, C., N. Dieleman, W.M. van der Flier, A. Versteeg, Y. Pijnenburg, P. Scheltens, F. Barkhof and H. Vrenken, More Atrophy of Deep Gray Matter Structures in Frontotemporal Dementia Compared to Alzheimer's Disease (2) 635-647

Monastero, R., see Bulati, M. (4) 1241-1251

Monastero, R., see Mangialasche, F. (2) 649-660

Monsch, A.U., see Egli, S.C. (2) 625-633

Moon, S.Y., see Park, H.K. (2) 661-673

Moran, R., see Nolan, J.M. (4) 1157-1169

Morbelli, S., A. Brugnolo, I. Bossert, A. Buschiazzo, G.B. Frisoni, S. Galluzzi, B.N.M. van Berckel, R. Ossenkoppele, R. Perneczky, A. Drzezga, M. Didic, E. Guedj, G. Sambuceti, G. Bottoni, D. Arnaldi, A. Picco, F. De Carli, M. Pagani and F.
Nobili, Visual Versus Semi-Quantitative Analysis of ${ }^{18}$ F-FDG-PET in Amnestic MCI: An European Alzheimer's Disease Consortium (EADC) Project (3) $815-826$

Moreno, J.M.R., see Naranjo, I.C. (2) 695-704

Moreno, S., see MacPherson, S.E. (2) 481-492

Morgan, A., see Claxton, A. (3) 897-906

Mori, S., see Nowrangi, M.A. (2) 585-598

Morikawa, M., see Okamoto, N. (3) 777-786

Morris, H.R., see Ryan, N.S. (4) 1069-10704

Morris, J., see Semrau, M. (1) 139-151

Mösch, E., see Ramirez, A. (4) 1203-1212

Mouchoux, C., see Dauphinot, V. (3) 907-916

Mowszowski, L., see Diamond, K. (4) 1181-1191

Mulcahy, R., see Nolan, J.M. (4) 1157-1169

Muller, M., see Jochemsen, H.M. (1) 153-162

Multhaup, G., see Barucker, C. (2) 613-624

Muñoz-Ruiz, M., see Hall, A. (1) 79-92

Murray, A.D., see Wischik, C.M. (2) 705-720

Na, D.L., see Kim, H.J. (3) 963-975

Na, D.L., see Kim, Y.J. (4) 1075-1085

Na, D.L., see Park, H.K. (2) 661-673

Na, H.R., see Park, H.K. (2) 661-673

Näätänen, R., see Stothart, G. (2) 397-408

Nagel, H., see Renziehausen, J. (3) 879-896

Naglie, G., see Charles, J. (2) 425-430

Naismith, S.L., see Diamond, K. (4) 1181-1191

Naismith, S.L., see Elcombe, E.L. (4) 1279-1290

Nakamura, A., see Cuesta, P. (2) 493-505

Naranjo, I.C., J.C.P. Cuenca, B.D. de San Juan, A.F. García, R.R. Sevilla, A.S. Cabrera, C.C. Hijón, S.R. Chala, J.M. Fuentes and J.M.R. Moreno, Association of Vascular Factors and Amnestic Mild Cognitive Impairment: A Comprehensive Approach (2) 695-704

Nasarauddin, M.B., see Graham, S.F. (3) $851-857$

Nathanson, L., see Humphries, C.E. (3) 977-987

Ng, O.T.-W., see Huang, C. (3) 839-850

Ng, T.P., see Goh, L.K. (1) 57-61

Ngandu, T., see Vuorinen, M. (1) 93-101

Nicholson, J.K., see Chang, K.L. (1) 215-231

Nicolia, V., see Persichilli, S. (4) 1323-1331

Nijpels, G., see Teunissen, C.E. (3) 809-813

Nilsson, S., see Kettunen, P. (4) 13131322

Niskanen, E., see Vuorinen, M. (1) 93-101

Nobili, F., see Morbelli, S. (3) 815-826

Noga, L., see Zimny, A. (1) 329-338

Nolan, J.M., E. Loskutova, A. Howard, R. Mulcahy, R. Moran, J. Stack, M. Bolger, R.F. Coen, J. Dennison, K.O. Akuffo, N. Owens, R. Power, D. Thurnham 
and S. Beatty, The Impact of Supplemental Macular Carotenoids in Alzheimer's Disease: A Randomized Clinical Trial (4) 1157-1169

Nordlund, A., see Eckerström, C. (1) 205-214

Norrie, L., see Diamond, K. (4) 1181-1191

Norrie, L., see Elcombe, E.L. (4) 1279-1290

Nowrangi, M.A., O. Okonkwo, C. Lyketsos, K. Oishi, S. Mori, M. Albert and M.M. Mielke, Atlas-Based Diffusion Tensor Imaging Correlates of Executive Function (2) 585-598

Ntovas, K., see Zygouris, S. (4) 1333-1347

O’Donnell, P., see Watt, A.D. (4) 1131-1143

O'Rourke, M., see Stone, J. (2) 355-373

Oh, H.G., see Chun, Y.S. (1) 261-275

Oishi, K., see Nowrangi, M.A. (2) 585-598

Okamoto, N., M. Morikawa, K. Tomioka, M. Yanagi, N. Amano and N. Kurumatani, Association between Tooth Loss and the Development of Mild Memory Impairment in the Elderly: The Fujiwarakyo Study (3) 777-786

Okonkwo, O., see Nowrangi, M.A. (2) 585-598

Oláh, Z., J. Kálmán, M.E. Tóth, Á. Zvara, M. Sántha, E. Ivitz, Z. Janka and M. Pákáski, Proteomic Analysis of Cerebrospinal Fluid in Alzheimer's Disease: Wanted Dead or Alive (4) 1303-1312

Olde Rikkert, M.G.M., F.R. Verhey, R. Blesa, C.A.F. von Arnim, A. Bongers, J. Harrison, J. Sijben, E. Scarpini, M.F.J. Vandewoude, B. Vellas, R. Witkamp, P.J.G.H. Kamphuis and P. Scheltens, Tolerability and Safety of Souvenaid in Patients with Mild Alzheimer's Disease: Results of MultiCenter, 24-Week, Open-Label Extension Study (2) 471-480

Olsson, E., see Eckerström, C. (1) 205-214

Olsson, S., see Kettunen, P. (4) 13131322

Ordóñez-Gutiérrez, L., M. Antón and F. Wandosell, Peripheral Amyloid Levels Present Gender Differences Associated with Aging in A $\beta P P / P S 1$ Mice (4) 1063-1068

Ossenkoppele, R., see Morbelli, S. (3) 815-826

Oudart, J.-B., see Salza, R. (4) 1253-1261

Owens, N., see Nolan, J.M. (4) 1157-1169

Pagani, M., see Morbelli, S. (3) 815-826

Pákáski, M., see Oláh, Z. (4) 1303-1312

Pal, A., M. Siotto, R. Prasad and R. Squitti, Towards a Unified Vision of Copper Involvement in Alzheimer's Disease: A Review Connecting Basic, Experimental, and Clinical Research (2) 343-354

Palumbo, C., see Semrau, M. (1) 139-151
Paolini, F., see Di Domenico, F., (4) 1107-1120

Paradise, M., see Diamond, K. (4) 1181-1191

Park, H.K., S.H. Choi, S.A. Park, H.J. Kim, Y. Lee, S.-H. Han, E.-J. Kim, B.C. Kim, H.J. Han, S.Y. Moon, D.W. Yang, K.W. Park, K.H. Park, B. Yoon, S.W. Seo, D.L. Na, H.R. Na and J.-H. Lee, Cognitive Profiles and Neuropsychiatric Symptoms in Korean Early-Onset Alzheimer's Disease Patients: A CREDOS Study (2) 661-673

Park, K.H., see Park, H.K. (2) 661-673

Park, K.W., see Park, H.K. (2) 661-673

Park, M.K., see Chun, Y.S. (1) 261-275

Park, S.A., see Park, H.K. (2) 661-673

Park, S.Y., see Jang, J.-W. (3) 1023-1034

Park, Y., see Chun, Y.S. (1) 261-275

Park, Y.H., see Jang, J.-W. (3) 1023-1034

Parra, M.A., see MacPherson, S.E. (2) 481-492

Parubrub, A.C., see Rueli, R.H.L.H. (2) 379-383

Passmore, A.P., see Graham, S.F. (3) 851-857

Pee, H.N., see Chang, K.L. (1) 215-231

Pentzek, M., see Ramirez, A. (4) 1203-1212

Perez, K., see Robb, E. (1) 69-78

Perez, K.A., see Watt, A.D. (4) 1131-1143

Pericak-Vance, M.A., see Humphries, C.E. (3) 977-987

Perluigi, M., see Di Domenico, F., (4) 1107-1120

Perneczky, R., see Morbelli, S. (3) 815-826

Perri, R., see Costa, A. (1) 63-67

Perry, G., see Bowen, R.L. (2) 549-560

Perry, G., Two Hundred Treasured Issues! (3) 725

Persichilli, S., J. Gervasoni, A. Di Napoli, A. Fuso, V. Nicolia, B. Giardina, S. Scarpa, C. Desiderio and R.A. Cavallaro, Plasma Thiols Levels in Alzheimer's Disease Mice under Diet-Induced Hyperhomocysteinemia: Effect of S-Adenosylmethionine and SuperoxideDismutase Supplementation (4) 1323-1331

Pertile, K.K., see Watt, A.D. (4) 1131-1143

Peters, O., see Barucker, C. (2) 613-624

Peterson, C., see Rembach, A. (3) 917-925

Petit, P.-D., see König, A. (2) 675-685

Pham, W., see McClure, R. (1) 283-295

Picco, A., see Morbelli, S. (3) 815-826

Pietroboni, A.M., see Calvi, A. (1) 277-282

Piguet, O., C.E. Leyton, L.D. Gleeson, C. Hoon and J.R. Hodges, Memory and Emotion Processing Performance Contributes to the Diagnosis of Non-Semantic Primary Progressive Aphasia Syndromes (2) 541-547

Pijnenburg, Y., see Möller, C. (2) 635-647

Pineda-Pardo, J.A., see Cuesta, P. (2) 493-505

Piobbico, D., see Mangialasche, F. (2) 649-660 
Poljak, A., see Lazarus, J. (1) 175-182

Polydoro, M., see Saidi, L.-J. (3) 937-947

Potter, W.Z., see Seeburger, J.L. (2) 525-539

Power, R., see Nolan, J.M. (4) 1157-1169

Prasad, R., see Pal, A. (2) 343-354

Preiss, R., see West, R.K. (3) 927-935

Prina, A.M., see Wu, Y.-T. (4) 1099-1106

Prokein, J., see Ramirez, A. (4) 1203-1212

Puig, K.L., B.M. Lutz, S.A. Urquhart, A.A. Rebel, X. Zhou, G.D. Manocha, M.A. Sens, A.K. Tuteja, N.L. Foster and C.K. Combs, Overexpression of Mutant Amyloid- $\beta$ Protein Precursor and Presenilin 1 Modulates Enteric Nervous System (4) 1263-1278

Pupo, G., see Di Domenico, F., (4) 1107-1120

Qu, Z., see Yu, J. (2) 455-469

Raji, C.A., see Riverol, M. (1) 319-328

Ramirez, A., S. Wolfsgruber, C. Lange, H. Kaduszkiewicz, S. Weyerer, J. Werle, M. Pentzek, A. Fuchs, S.G. Riedel-Heller, T. Luck, E. Mösch, H. Bickel, B. Wiese, J. Prokein, H.-H. König, C. Brettschneider, M.M. Breteler, W. Maier, F. Jessen, M. Scherer and for the AgeCoDe Study Group, Elevated $\mathrm{HbA}_{1 \mathrm{c}}$ is Associated with Increased Risk of Incident Dementia in Primary Care Patients (4) 1203-1212

Ramont, L., see Salza, R. (4) 1253-1261

Rangaraju, S., M. Gearing, L.-W. Jin and A. Levey, Potassium Channel Kv1.3 Is Highly Expressed by Microglia in Human Alzheimer's Disease (3) 797-808

Ravona-Springer, R., S. Haratz, D. Tanne, J. Schmeidler, S. Efrati, C. Rosendorff, M.S. Beeri and J.M. Silverman, Arterial Wall Function is Associated with Cognitive Performance Primarily in Elderly with Type 2 Diabetes (2) 687-693

Ravona-Springer, R., see West, R.K. (3) 927-935

Rebel, A.A., see Puig, K.L. (4) 1263-1278

Regeniter, A., see Egli, S.C. (2) 625-633

Rembach, A., F.C. Stingo, C. Peterson, M. Vannucci, K.-A. Do, W.J. Wilson, S.L. Macaulay, T.M. Ryan, R.N. Martins, D. Ames, C.L. Masters, J.D. Doecke and the AIBL Research Group, Bayesian Graphical Network Analyses Reveal Complex Biological Interactions Specific to Alzheimer's Disease (3) 917-925

Rembach, A., see Watt, A.D. (4) 1131-1143

Renziehausen, J., C. Hiebel, H. Nagel, A. Kundu, S. Kins, D. Kögel, C. Behl and P. Hajieva, The
Cleavage Product of Amyloid- $\beta$ Protein Precursor sA $\beta$ PP $\alpha$ Modulates BAG3-Dependent Aggresome Formation and Enhances Cellular Proteasomal Activity (3) 879-896

Revesz, T., see Ryan, N.S. (4) 1069-10704

Ribeiro, C., see Barrett, J.P. (3) 949-962

Ricard-Blum, S., see Salza, R. (4) 1253-1261

Riedel-Heller, S.G., see Ramirez, A. (4) 1203-1212

Riedinger, J.-M., see Zarrouk, A. (4) 1349-1359

Rikkert, M.O., see Semrau, M. (1) 139-151

Rissman, R.A., see Zhang, C. (2) 375-378

Rist, P.M., J.R. Marden, B.D. Capistrant, Q. Wu and M.M. Glymour, Do Physical Activity, Smoking, Drinking, or Depression Modify Transitions from Cognitive Impairment to Functional Disability? (4) 1171-1180

Riverol, M., J.T. Becker, O.L. López, C.A. Raji, P.M. Thompson, O.T. Carmichael, H.M. Gach, W.T. Longstreth Jr., L. Fried, R.P. Tracy and L.H. Kuller, Relationship between Systemic and Cerebral Vascular Disease and Brain Structure Integrity in Normal Elderly Individuals (1) 319-328

Robb, E., K. Perez, L.W. Hung, C.L. Masters, K.J. Barnham, R.A. Cherny, A.I. Bush, P.A. Adlard and D.I. Finkelstein, High Order W02-Reactive Stable Oligomers of Amyloid- $\beta$ are Produced in vivo and in vitro via Dialysis and Filtration of Synthetic Amyloid- $\beta$ Monomer (1) 69-78

Robert, P., see König, A. (2) 675-685

Robert, P., see Semrau, M. (1) 139-151

Romano III, R.R., see Gifford, K.A. (1) 309-318

Rosendorff, C., see Ravona-Springer, R. (2) 687-693

Rouaud, O., see Zarrouk, A. (4) 1349-1359

Rouch, I., see Dauphinot, V. (3) 907-916

Rowe, C.C., see Woodward, M.C. (1) 233-242

Royse, A.G., see Callaway, J.K. (3) 995-1005

Royse, C.F., see Callaway, J.K. (3) 995-1005

Ruberg, F.L., see Jefferson, A.L. (4) 1361-1373

Rüegger-Frey, B., see Suppa, P. (1) 183-193

Rueli, R.H.L.H., A.C. Parubrub, A.S.T. Dewing, A.C. Hashimoto, M.T. Bellinger, E.J. Weeber, J.H. Uyehara-Lock, L.R. White, M.J. Berry and F.P. Bellinger, Increased Selenoprotein $\mathrm{P}$ in Choroid Plexus and Cerebrospinal Fluid in Alzheimer's Disease Brain (2) 379-383

Ruggiero, C., see Mangialasche, F. (2) 649-660

Rumble, R.L., see Watt, A.D. (4) 1131-1143

Ryan, N.S., T. Lashley, T. Revesz, K. Dantu, N.C. Fox and H.R. Morris, Spontaneous ARIA (AmyloidRelated Imaging Abnormalities) and Cerebral Amyloid Angiopathy Related Inflammation in 
Presenilin 1-Associated Familial Alzheimer's Disease (4) 1069-10704

Ryan, T.M., see Rembach, A. (3) 917-925

Sachdev, P.S., see Lazarus, J. (1) 175-182

Saidi, L.-J., M. Polydoro, K.R. Kay, L. Sanchez, E.-M. Mandelkow, B.T. Hyman and T.L. Spires-Jones, Carboxy Terminus Heat Shock Protein 70 Interacting Protein Reduces Tau-Associated Degenerative Changes (3) 937-947

Sala, S.D., see MacPherson, S.E. (2) 481-492

Salomaa, V., see Tynkkynen, J. (3) 1007-1013

Salza, R., J.-B. Oudart, L. Ramont, F.-X. Maquart, S. Bakchine, H. Thoannès and S. Ricard-Blum, Endostatin Level in Cerebrospinal Fluid of Patients with Alzheimer's Disease (4) 1253-1261 Sambuceti, G., see Morbelli, S. (3) 815-826

Samuels, L.R., see Gifford, K.A. (1) 309-318 Sanchez, L., see Saidi, L.-J. (3) 937-947

Sancho, M., see Cuesta, P. (2) 493-505

Sántha, M., see Oláh, Z. (4) 1303-1312

Sartorius, N., see Semrau, M. (1) 139-151

Sasiadek, M., see Zimny, A. (1) 329-338

Savage, M.J., see Seeburger, J.L. (2) 525-539

Scarpa, S., see Persichilli, S. (4) 1323-1331

Scarpini, E., see Calvi, A. (1) 277-282

Scarpini, E., see Olde Rikkert, M.G.M. (2) 471-480

Schachtman, T.R., see Walker, J.M. (2) 561-572

Scheltens, P., see Jochemsen, H.M. (1) 153-162

Scheltens, P., see Möller, C. (2) 635-647

Scheltens, P., see Olde Rikkert, M.G.M. (2) 471-480

Scheltens, P., see Teunissen, C.E. (3) 809-813

Scherer, M., see Ramirez, A. (4) 1203-1212

Schipke, C.G., see Barucker, C. (2) 613-624

Schmeidler, J., see Ravona-Springer, R. (2) 687-693

Schmeidler, J., see West, R.K. (3) 927-935

Schmitt, F.A., see Di Domenico, F., (4) 1107-1120

Seeburger, J.L., D.J. Holder, M. Combrinck, C. Joachim, O. Laterza, M. Tanen, A. Dallob, D. Chappell, K. Snyder, M. Flynn, A. Simon, V. Modur, W.Z. Potter, G. Wilcock, M.J. Savage and A.D. Smith, Cerebrospinal Fluid Biomarkers Distinguish Postmortem-Confirmed Alzheimer's Disease from Other Dementias and Healthy Controls in the OPTIMA Cohort (2) 525-539

Segkouli, S., see Zygouris, S. (4) 1333-1347

Semrau, M., A. Burns, S. Djukic-Dejanovic, D. Eraslan, C. Han, D. Lecic-Tosevski, A. Lobo, A. Mihai, J. Morris, C. Palumbo, P. Robert, G. Stiens, G. Stoppe, U. Volpe, M.O. Rikkert and N. Sartorius on behalf of the International Dementia Alliance
(IDEAL) study group, Development of an International Schedule for the Assessment and Staging of Care for Dementia (1) 139-151

Sens, M.A., see Puig, K.L. (4) 1263-1278

Seo, S.W. see Kim, Y.J. (4) 1075-1085

Seo, S.W., see Kim, H.J. (3) 963-975

Seo, S.W., see Park, H.K. (2) 661-673

Seong, J.-K. see Kim, Y.J. (4) 1075-1085

Seppi, D., see Koal, T. (4) 1193-1201

Serfozo, P., see Walker, J.M. (2) 561-572

Sergio, L.E., see Hawkins, K.M. (3) 867-878

Serpente, M., see Calvi, A. (1) 277-282

Serra, L., see Bozzali, M. (1) 243-250

Sevilla, R.R., see Naranjo, I.C. (2) 695-704

Shafagoj, Y., see Al-khateeb, E. (3) 859-865

Sharp, F.R., see Zhan, X. (4) 1213-1229

Shih, Y.-C., see Chang, Y.-L. (1) 125-138

Shimohama, S., see Matsumura, A. (2) 409-423

Sieczkowski, E., I. Milenkovic, V. Venkataramani, R. Giera, T. Ströbel, R. Höftberger, P.P. Liberski, E. Auff, O. Wirths, T.A. Bayer and G.G. Kovacs, I716F $A \beta P P$ Mutation Associates with the Deposition of Oligomeric Pyroglutamate Amyloid- $\beta$ and $\alpha$-Synucleinopathy with Lewy Bodies (1) 103-114

Sijben, J., see Olde Rikkert, M.G.M. (2) 471-480

Silverman, J.M., see Ravona-Springer, R. (2) 687-693

Silverman, J.M., see West, R.K. (3) 927-935

Simon, A., see Seeburger, J.L. (2) 525-539

Simonyi, A., see Walker, J.M. (2) 561-572

Siotto, M., see Pal, A. (2) 343-354

Sjölander, A., see Kettunen, P. (4) 13131322

Skinner, J.S., see Jefferson, A.L. (4) 1361-1373

Smith, A.D., see Seeburger, J.L. (2) 525-539

Smith, M.A. see Bowen, R.L. (2) 549-560

Snyder, K., see Seeburger, J.L. (2) 525-539

Soininen, H., see Hall, A. (1) 79-92

Soininen, H., see Vuorinen, M. (1) 93-101

Sollberger, M., see Egli, S.C. (2) 625-633

Solomon, A., see Vuorinen, M. (1) 93-101

Sommer, A., see Barucker, C. (2) 613-624

Song, F., see Lazarus, J. (1) 175-182

Spanò, B., see Bozzali, M. (1) 243-250

Sparling, J., see Jefferson, A.L. (4) 1361-1373

Spies, L., see Suppa, P. (1) 183-193

Spires-Jones, T.L., see Saidi, L.-J. (3) 937-947

Spulber, G., see Vuorinen, M. (1) 93-101

Squitti, R., see Pal, A. (2) 343-354

St George-Hyslop, P., see Barucker, C. (2) 613-624

Stack, J., see Nolan, J.M. (4) 1157-1169

Staff, R.T., see Wischik, C.M. (2) 705-720

Stamova, B., see Zhan, X. (4) 1213-1229 
Stanwood, G., see McClure, R. (1) 283-295

Stec, D., see McClure, R. (1) 283-295

Stiens, G., see Semrau, M. (1) 139-151

Stingo, F.C., see Rembach, A. (3) 917-925

Stone, J., D.M. Johnstone, J. Mitrofanis and M. O'Rourke, The Mechanical Cause of Age-Related Dementia (Alzheimer's Disease): The Brain is Destroyed by the Pulse (2) 355-373

Stoppe, G., see Semrau, M. (1) 139-151

Storey, J.M.D., see Wischik, C.M. (2) 705-720

Stothart, G., N. Kazanina, R. Näätänen, J. Haworth and A. Tales, Early Visual Evoked Potentials and Mismatch Negativity in Alzheimer's Disease and Mild Cognitive Impairment (2) 397-408

Ströbel, T., see Sieczkowski, E. (1) 103-114

Suain, V., see Frederick, C. (4) 1145-1156

Sumner, E.C., see Jefferson, A.L. (4) 1361-1373

Sun, G.Y., see Walker, J.M. (2) 561-572

Suppa, P., U. Anker, L. Spies, I. Bopp, B. Rüegger-Frey, R. Klaghofer, C. Gocke, H. Hampel, S. Beck and R. Buchert, Fully Automated Atlas-Based Hippocampal Volumetry for Detection of Alzheimer's Disease in a Memory Clinic Setting (1) 183-193

Suzuki, H., see Matsumura, A. (2) 409-423

Suzuki, S., see Matsumura, A. (2) 409-423

Szewczyk, P., see Zimny, A. (1) 329-338

Takata, K., see Matsumura, A. (2) 409-423

Tales, A., see Stothart, G. (2) 397-408

Tamburin, S., see Testi, S. (1) 49-56

Tan, C.-C., see Wang, J. (2) 385-396

Tan, C.-C., see Zhu, X.-C. (1) 163-174

Tan, C.H., see Goh, L.K. (1) 57-61

Tan, L., see Wang, H.-F. (1) 115-123

Tan, L., see Wang, J. (2) 385-396

Tan, L., see Zhu, X.-C. (1) 163-174

Tan, M.-S., see Wang, H.-F. (1) 115-123

Tan, W.P., see Chang, K.L. (1) 215-231

Tanen, M., see Seeburger, J.L. (2) 525-539

Tang, S.-W., see Wang, J. (2) 385-396

Tang, X., D. Holland, A.M. Dale, L. Younes, M.I. Miller and for the Alzheimer's Disease Neuroimaging Initiative, Baseline Shape Diffeomorphometry Patterns of Subcortical and Ventricular Structures in Predicting Conversion of Mild Cognitive Impairment to Alzheimer's Disease (2) 599-611

Tanne, D., see Ravona-Springer, R. (2) 687-693

Tay, L., see Goh, L.K. (1) 57-61

Taylor, K.I., see Egli, S.C. (2) 625-633

Teipel, S., see Berger, C. (2) 439-453

Teipel, S., see Wucherer, D. (3) 1015-1021
Teo, S., see Goh, L.K. (1) 57-61

Testi, S., S. Tamburin, G. Zanette and G.M. Fabrizi, Co-Occurrence of the C9ORF72 Expansion and a Novel GRN Mutation in a Family with Alternative Expression of Frontotemporal Dementia and Amyotrophic Lateral Sclerosis (1) 49-56

Teunissen, C.E., see Jochemsen, H.M. (1) 153-162

Teunissen, C.E., W.M. van der Flier, P. Scheltens, A. Duits, N. Wijnstok, G. Nijpels, J.M. Dekker, M.A. Blankenstein and A.C. Heijboer, Serum Leptin is not Altered nor Related to Cognitive Decline in Alzheimer's Disease (3) 809-813

Thalamuthu, A., see Lazarus, J. (1) 175-182

Thoannès, H., see Salza, R. (4) 1253-1261

Thomas, A.J., see Khundakar, A.A. (1) 27-41

Thompson, P.M., see Riverol, M. (1) 319-328

Thurnham, D., see Nolan, J.M. (4) 1157-1169

Thyrian, J.R., see Wucherer, D. (3) 1015-1021

Tierney, M.C., see Charles, J. (2) 425-430

Tomioka, K., see Okamoto, N. (3) 777-786

Tooyama, I., see McClure, R. (1) 283-295

Torso, M., see Bozzali, M. (1) 243-250

Tóth, M.E., see Oláh, Z. (4) 1303-1312

Tracy, R.P., see Riverol, M. (1) 319-328

Trittschuh, E.H., see Claxton, A. (3) 897-906

Trounson, B.O., see Watt, A.D. (4) 1131-1143

Trypka, E., see Zimny, A. (1) 329-338

Tsolaki, M., see Hall, A. (1) 79-92

Tsolaki, M., see Zygouris, S. (4) 1333-1347

Tuteja, A.K., see Puig, K.L. (4) 1263-1278

Tynkkynen, J., T. Laatikainen, V. Salomaa, A.S. Havulinna, S. Blankenberg, T. Zeller and J.A. Hernesniemi, NT-proBNP and the Risk of Dementia: A Prospective Cohort Study with 14 Years of Follow-Up (3) $1007-1013$

Tzovaras, D., see Zygouris, S. (4) 1333-1347

Urquhart, S.A., see Puig, K.L. (4) 1263-1278

Uyehara-Lock, J.H., see Rueli, R.H.L.H. (2) 379-383

van Berckel, B.N.M., see Morbelli, S. (3) 815-826

van der Flier, W.M., see Jochemsen, H.M. (1) 153-162

van der Flier, W.M., see Möller, C. (2) 635-647

van der Flier, W.M., see Teunissen, C.E. (3) 809-813

Vandewoude, M.F.J., see Olde Rikkert, M.G.M. (2) 471-480

Vannucci, M., see Rembach, A. (3) 917-925

Varos, T.A., see Woodward, M.C. (1) 233-242

Vellas, B., see Hall, A. (1) 79-92

Vellas, B., see Olde Rikkert, M.G.M. (2) 471-480

Venkataramani, V., see Sieczkowski, E. (1) 103-114 
Verhey, F., see König, A. (2) 675-685

Verhey, F.R., see Olde Rikkert, M.G.M. (2) 471-480

Versteeg, A., see Möller, C. (2) 635-647

Vijayaraghavan, A., see Goh, L.K. (1) 57-61

Villard, V., see Meunier, J. (2) 507-524

Villemagne, V.L., see Watt, A.D. (4) 1131-1143

Villemagne, V.L., see Woodward, M.C. (1) 233-242

Visser, P.J., see Hall, A. (1) 79-92

Volpe, U., see Semrau, M. (1) 139-151

von Arnim, C.A.F., see Olde Rikkert, M.G.M. (2) 471480

Votis, K., see Zygouris, S. (4) 1333-1347

Vrenken, H., see Möller, C. (2) 635-647

Vuorinen, M., G. Spulber, S. Damangir, E. Niskanen, T. Ngandu, H. Soininen, M. Kivipelto and A. Solomon, Midlife CAIDE Dementia Risk Score and Dementia-Related Brain Changes up to 30 Years Later on Magnetic Resonance Imaging (1) 93-101

Walker, J.M., D. Klakotskaia, D. Ajit, G.A. Weisman, W.G. Wood, G.Y. Sun, P. Serfozo, A. Simonyi and T.R. Schachtman, Beneficial Effects of Dietary EGCG and Voluntary Exercise on Behavior in an Alzheimer's Disease Mouse Model (2) 561-572

Wallin, A., see Eckerström, C. (1) 205-214

Wandosell, F., see Ordóñez-Gutiérrez, L. (4) 1063-1068 Wang, C., see Wang, J. (2) 385-396

Wang, C., see Zhu, X.-C. (1) 163-174

Wang, H.-F., L. Tan, X.-K. Hao, T. Jiang, M.-S. Tan, Y. Liu, D.-Q. Zhang, J.-T. Yu and for the Alzheimer's Disease Neuroimaging Initiative, Effect of EPHA1 Genetic Variation on Cerebrospinal Fluid and Neuroimaging Biomarkers in Healthy, Mild Cognitive Impairment and Alzheimer's Disease Cohorts (1) 115-123

Wang, H.-F., see Wang, J. (2) 385-396

Wang, H.-F., see Zhu, X.-C. (1) 163-174

Wang, J., L. Tan, H.-F. Wang, C.-C. Tan, X.-F. Meng, C. Wang, S.-W. Tang and J.-T. Yu, Anti-Inflammatory Drugs and Risk of Alzheimer's Disease: An Updated Systematic Review and Meta-Analysis (2) 385-396

Wang, J., see Zhu, X.-C. (1) 163-174

Wang, X., see Chaudhry, M. (3) 989-994

Watt, A.D., K.A. Perez, C.-S. Ang, P. O’Donnell, A. Rembach, K.K. Pertile, R.L. Rumble, B.O. Trounson, C.J. Fowler, N.G. Faux, C.L. Masters, V.L. Villemagne and K.J. Barnham, Peripheral $\alpha$-Defensins 1 and 2 are Elevated in Alzheimer's Disease (4) 1131-1143

Wattjes, M.P., see Jochemsen, H.M. (1) 153-162
Weeber, E.J., see Rueli, R.H.L.H. (2) 379-383

Weggen, S., see Hüttenrauch, M. (4) 1291-1302

Weinshenker, D., see Liu, X. (1) 251-260

Weisman, G.A., see Walker, J.M. (2) 561-572

Werle, J., see Ramirez, A. (4) 1203-1212

West, R.K., R. Ravona-Springer, A. Heymann, J. Schmeidler, D. Leroith, K. Koifman, E. Guerrero-Berroa, R. Preiss, H. Hoffman, J.M. Silverman and M.S. Beeri, Shorter Adult Height is Associated with Poorer Cognitive Performance in Elderly Men with Type II Diabetes (3) 927-935

Weyerer, S., see Ramirez, A. (4) 1203-1212

White, L.R., see Rueli, R.H.L.H. (2) 379-383

Whitehead, P., see Humphries, C.E. (3) 977-987

Wiese, B., see Ramirez, A. (4) 1203-1212

Wijnstok, N., see Teunissen, C.E. (3) 809-813

Wilcock, G., see Seeburger, J.L. (2) 525-539

Williams, P.T., Lower Risk of Alzheimer's Disease Mortality with Exercise, Statin, and Fruit Intake (4) 1121-1129

Wilson, W.J., see Rembach, A. (3) 917-925

Winchester, L., see Kawada, H. (1) 297-307

Wirths, O., see Hüttenrauch, M. (4) 1291-1302

Wirths, O., see Sieczkowski, E. (1) 103-114

Wischik, C.M., R.T. Staff, D.J. Wischik, P. Bentham, A.D. Murray, J.M.D. Storey, K.A. Kook and C.R. Harrington, Tau Aggregation Inhibitor Therapy: An Exploratory Phase 2 Study in Mild or Moderate Alzheimer's Disease (2) 705-720

Wischik, D.J., see Wischik, C.M. (2) 705-720

Witkamp, R., see Olde Rikkert, M.G.M. (2) 471-480

Wojtynska, R., see Zimny, A. (1) 329-338

Wolfsgruber, S., see Ramirez, A. (4) 1203-1212

Wong, G.T.-C., see Huang, C. (3) 839-850

Wood, W.G., see Walker, J.M. (2) 561-572

Woodward, M.C., C.C. Rowe, G. Jones, V.L. Villemagne and T.A. Varos, Differentiating the Frontal Presentation of Alzheimer's Disease with FDGPET (1) 233-242

Woolman, T., see Kawada, H. (1) 297-307

Wright, M.J., see Lazarus, J. (1) 175-182

Wu, Q., see Rist, P.M. (4) 1171-1180

Wu, Y.-T., W.B. Grant, A.M. Prina, H.-y. Lee and C. Brayne, Nutrition and the Prevalence of Dementia in Mainland China, Hong Kong, and Taiwan: An Ecological Study (4) 1099-1106

Wucherer, D., T. Eichler, I. Kilimann, J. Hertel, B. Michalowsky, J.R. Thyrian, S. Teipel and W. Hoffmann, Antidementia Drug Treatment in People Screened Positive for Dementia in Primary Care (3) $1015-1021$ 
Xhillari, D., see McClure, R. (1) 283-295

Xiong, C., see Bowen, R.L. (2) 549-560

Xu, H., see Yu, J. (2) 455-469

Yamauchi, A., see Matsumura, A. (2) 409-423

Yan, S.-H., see Chang, Y.-L. (1) 125-138

Yanagi, M., see Okamoto, N. (3) 777-786

Yanagisawa, D., see McClure, R. (1) 283-295

Yang, D.W., see Park, H.K. (2) 661-673

Yang, H.O., see Chun, Y.S. (1) 261-275

Yang, X., see Yu, J. (2) 455-469

Ye, B.S., see Kim, H.J. (3) 963-975

Ye, B.S., see Kim, Y.J. (4) 1075-1085

Ye, K., see Liu, X. (1) 251-260

Yoon, B., see Park, H.K. (2) 661-673

Youn, Y.C. see Jang, J.-W. (3) 1023-1034

Younes, L., see Tang, X. (2) 599-611

Yu, J., X. Luo, H. Xu, Q. Ma, J. Yuan, X. Li, R.C.-C. Chang, Z. Qu, X. Huang, Z. Zhuang, J. Liu and X. Yang, Identification of the Key Molecules Involved in Chronic Copper Exposure-Aggravated Memory Impairment in Transgenic Mice of Alzheimer's Disease Using Proteomic Analysis (2) 455-469

Yu, J.-T., see Wang, H.-F. (1) 115-123

Yu, J.-T., see Wang, J. (2) 385-396

Yu, J.-T., see Zhu, X.-C. (1) 163-174

Yu, S.-Y., T.-J. Lee, S.-H. Jang, J.W. Han, T.H. Kim and K.W. Kim, Cost-Effectiveness of Nationwide Opportunistic Screening Program for Dementia in South Korea (1) 195-204

Yu, Y., see Zhu, X.-C. (1) 163-174

Yuan, J., see Yu, J. (2) 455-469

Zanette, G., see Testi, S. (1) 49-56

Zarrouk, A., J.-M. Riedinger, S.H. Ahmed, S. Hammami, W. Chaabane, M. Debbabi, S.B. Ammou, O. Rouaud, M. Frih, G. Lizard and M. Hammami, Fatty Acid Profiles in Demented
Patients: Identification of Hexacosanoic Acid (C26:0) as a Blood Lipid Biomarker of Dementia (4) 1349-1359

Zelin, M.A., see Zhan, X. (4) 1213-1229

Zeller, T., see Tynkkynen, J. (3) 1007-1013

Zetterberg, H., see Kettunen, P. (4) 13131322

Zhan, X., G.C. Jickling, B.P. Ander, B. Stamova, D. Liu, P.F. Kao, M.A. Zelin, L.-W. Jin, C. DeCarli and F.R. Sharp, Myelin Basic Protein Associates with $A \beta P P, A \beta_{1-42}$, and Amyloid Plaques in Cortex of Alzheimer's Disease Brain (4) 1213-1229

Zhang, C., R.A. Rissman and J. Feng, Characterization of ATP Alternations in an Alzheimer's Disease Transgenic Mouse Model (2) 375-378

Zhang, D.-Q., see Wang, H.-F. (1) 115-123

Zhao, X., see Ji, L. (1) 13-25

Zhou, X., see Puig, K.L. (4) 1263-1278

Zhu, X.-C., Y. Yu, H.-F. Wang, T. Jiang, L. Cao, C. Wang, J. Wang, C.-C. Tan, X.-F. Meng, L. Tan and J.-T. Yu, Physiotherapy Intervention in Alzheimer's Disease: Systematic Review and MetaAnalysis (1) 163-174

Zhuang, Z., see Yu, J. (2) 455-469

Zimny, A., J. Bladowska, A. Macioszek, P. Szewczyk, E. Trypka, R. Wojtynska, L. Noga, J. Leszek and M. Sasiadek, Evaluation of the Posterior Cingulate Region with FDG-PET and Advanced MR Techniques in Patients with Amnestic Mild Cognitive Impairment: Comparison of the Methods (1) 329-338

Zvara, Á., see Oláh, Z. (4) 1303-1312

Zygouris, S., D. Giakoumis, K. Votis, S. Doumpoulakis, K. Ntovas, S. Segkouli, C. Karagiannidis, D. Tzovaras and M. Tsolaki, Can a Virtual Reality Cognitive Training Application Fulfill a Dual Role? Using the Virtual Supermarket Cognitive Training Application as a Screening Tool for Mild Cognitive Impairment (4) 1333-1347 